\title{
Identification of grain and flour quality determinants in common wheat using GWAS
}

\author{
Kiseleva A.A. ${ }^{1 *}$, Leonova I.N. ${ }^{1}$, Pshenichnikova T.A. ${ }^{1}$, Likhenko I.E. ${ }^{2}$, Ageeva E.V. ${ }^{2}$, \\ Stepochkina N.I. ${ }^{2}$, Salina E.A. ${ }^{1}$ \\ ${ }^{1}$ Institute of Cytology and Genetics, SB RAS, Novosibirsk, Russia \\ ${ }^{2}$ Siberian Research Institute of Plant Production and Breeding - Branch of the Institute of Cytology \\ and Genetics, SB RAS, Krasnoobsk, Novosibirsk region, Russia \\ *e-mail: antkiseleva@bionet.nsc.ru
}

In this study we investigated common wheat varieties adapted to the environment of Western Siberia and the Ural. The qualitative characteristics of flour and grain determine the technological properties of the product and its nutritional quality. The decrease of grain baking parameters result in the inability to produce a sufficient amount of good quality bread. Thus, it is necessary to identify new resources to improve these traits. We analyzed grain quality traits, such as nitrogen, protein, thousand grain weight, grain bulk density, vitrescence, falling number, gluten; and flour quality traits: flour particle diameter, flour surface, flour strength, dough resilience, dough strength and extensibility. The population of 92 common wheat varieties was genotyped using the $15 \mathrm{~K}$ Illumina Infinium SNP array (TraitGenetics GmbH). We used Mixed Linear Model implemented in EMMAX algorithm of R package GENESIS to perform association analysis. The results revealed SNP markers significantly associated with every trait. Some of the detected loci corresponded to known loci, but the others were firstly described. The results obtained will be tested in repeated study. Using the SNP sequences associated with the traits, markers will be developed to use them in further selection process.

Acknowledgements: This study was supported by the Russian Science Foundation Project No. 16-16-00011-P. 